Sandra Verhagen

Peter J. G. Teunissen

\section{On the probability density function of the GNSS ambiguity residuals}

probabilistic properties of the parameters involved. In this contribution we will present and evaluate the joint probability density function (PDF) of the multivariate integer GPS carrier phase ambiguity residuals. The residuals and their properties depend on the integer estimation principle used. Since it is known that the integer least-squares estimator is the optimal choice from the class of admissible integer estimators, we will only focus on the PDF of the ambiguity residuals for this estimator. Unfortunately the PDF cannot be evaluated exactly. It will therefore be shown how to obtain a good approximation. The evaluation will be completed by some examples. measure the significance of the methods are often based on incorrect assumptions with respect to the
Abstract Integer GNSS ambiguity validation of the unknown integer carrier phase ambiguities. A problem then is that the classical theor to the integer GPS model, and hence when use is made of the classical the integer GPS model is to ma quantify the inconsistency between data and model, while their proba-
S. Verhagen $(\bowtie)$ · P. J. G. Teunissen Space Systems, Delft University of Technology, Kluyverweg 1 , 2629 HS Delft, The Netherlands

E-mail: S.Verhagen@LR.TUDelft.nl
Received: 7 February 2005

Published online: 13 July 2005

\section{Integer ambiguity resolution}

The general GNSS observation model can be written in the form:

$y=A a+B b+e, \quad Q_{y}$

where $y$ is the random vector with $m$ double difference code and phase observations, $a$ the $n$-vector with unknown integer carrier phase ambiguities, i.e. $a \in Z^{n}, b$ is a $p$-vector with the unknown real-valued parameters, and $e$ is the noise vector. The real-valued parameters are referred to as the baseline unknowns, although $b$ may also contain for example atmospheric delays. The covariance matrix of the observation vector is given by $Q_{y}$.

Taking into account the integer nature of the ambiguity parameters in an optimal way, cf. (Teunissen 1999), involves that a non-standard least-squares problem has to be solved, referred to as integer leastsquares (ILS), (Teunissen 1993). The solution of model (1) is then obtained by the following minimization problem:

$\min _{a, b}\|y-A a-B b\|_{Q_{y}}^{2}, a \in Z^{n}, b \in R^{p}$ 
where $\|\cdot\|_{O}^{2}=(\cdot)^{T} Q^{-1}(\cdot)$. The following orthogonal decomposition can be used:

$$
\|y-A a-B b\|_{Q_{y}}^{2}=\|\hat{e}\|_{Q_{y}}^{2}+\|\hat{a}-a\|_{Q_{\hat{a}}}^{2}+\|\hat{b}(a)-b\|_{Q_{\hat{b} \mid \hat{a}}}^{2}
$$

with the residual estimator $\hat{e}=y-A \hat{a}-B \hat{b}$, the conditional baseline estimator $\hat{b}(a)=\hat{b}-Q_{\hat{b} \hat{a}} Q_{\hat{a}}^{-1}(\hat{a}-a)$, and corresponding covariance matrix $Q_{\hat{b} \mid \hat{a}}=Q_{\hat{b}}-$ $Q_{\hat{b} \hat{a}} Q_{\hat{a}}^{-1} Q_{\hat{a} \hat{b}}$.

It follows from Eq. 3 that the solution of the minimization problem in Eq. 2 is obtained using a three step procedure. The unconstrained least-squares solution is referred to as the float solution, with estimators $\hat{a}$ and $\hat{b}$, and residual vector $\hat{e}$. Taking into account the integer nature of the ambiguities using integer least-squares, means that the second term on the right-hand side of Eq. 3 needs to be minimized. This is the ambiguity resolution step, providing the fixed ambiguities $\vec{a}$. Finally, solving for the last term corresponds to fixing the baseline, $\vec{b}=\hat{b}-Q_{\hat{b} \hat{a}} Q_{\hat{a}}^{-1}(\hat{a}-\bar{a})$, so that the last term in Eq. 3 becomes zero, and the minimization problem of Eq. 2 is indeed solved.

The ambiguity resolution step involves a mapping from the $n$-dimensional space of reals to the $n$ dimensional space of integers. In the integer leastsquares approach a subset $S_{z} \subset R^{n}$ is assigned to each integer vector $z \in Z^{n}$. This subset is called the pull-in region and is defined as the collection of all $x \in R^{n}$ that are closer to $z$ than to any other integer grid point in $R^{n}$, where the distance is measured in the metric of $Q_{\hat{a}}$.

In this contribution it will be assumed that integer least-squares is used for ambiguity resolution. As mentioned above, this will result in the optimal solution in the sense that the probability of correct integer estimation is maximized, cf. (Teunissen 1999). An efficient implementation of the integer least-squares principle is the LAMBDA method, (Teunissen 1993; Teunissen 1995; De Jonge and Tiberius 1996). Examples of the use of the LAMBDA method in practice can be found e.g. in (Boon and Ambrosius, 1997; Cox and Brading, 1999 and Peng, Chang and Wang, 1999).

However, a parameter estimation theory cannot be considered complete without the appropriate measures to validate the parameter solution. Parameter estimation provides the estimates of the unknown parameters, together with the corresponding covariance matrices. In the classical theory of linear estimation, the covariance matrices provide sufficient information on the precision of the estimated parameters. The reason is that a linear model applied to normally (Gaussian) distributed data, provides linear estimators that are also normally distributed, and the peakedness of the multivariate normal distribution is completely captured by the covariance matrix. The more peaked the PDF is, the higher the precision is.

Unfortunately, this relatively simple approach cannot be applied in case integer parameters are involved in the estimation process, since the integer estimators do not have a Gaussian distribution, even if the model is linear and the data are normally distributed. Instead of the covariance matrices, we therefore have to use the parameter distribution itself in order obtain the appropriate measures that can be used to validate the integer parameter solution. For that purpose, the probability density function (PDF) of the ambiguity residuals can be used, since the residuals quantify the inconsistency between data and model, while the PDF describes their probabilistic properties, which are a measure for the significance of the inconsistency.

In Teunissen $(2003 \mathrm{a}$; b) the new class of integer aperture estimators is introduced. The integer aperture ambiguity estimator, $\bar{a}$, is defined as follows:

$\bar{a}= \begin{cases}\breve{a} & \text { if } \hat{a} \in \Omega=\bigcup_{z \in Z^{n}} \Omega_{z}, \Omega_{z} \subset S_{z} \\ \hat{a} & \text { otherwise }\end{cases}$

So, the integer aperture estimate equals the fixed estimate if and only if the float ambiguity vector is an element of the so-called aperture space, $\Omega$, which is built up of translational invariant aperture pull-in regions $\Omega_{z}$ $\subset S_{z}, S_{z}=S_{0}+z$. Different estimators can be defined within this class by choosing the 'shape' of the aperture pull-in regions. Based on this class for the first time it is possible to define a theoretically sound and overall approach to the problem of integer estimation and validation. This is possible by using the fixed fail rate approach: the user specifies the maximum allowable probability of incorrect fixing (the fail rate), and based on this choice the size of the aperture pull-in regions is determined.

In Teunissen $(2004 ; 2005)$ two important examples of integer aperture estimators have been presented, namely the Penalized Integer Aperture estimator and the Optimal Integer Aperture estimator, respectively. The latter is optimal in the sense that the success rate is maximized for a fixed fail rate. The probabilistic evaluation of both estimators requires the knowledge of the PDF of the ambiguity residuals, $\breve{\varepsilon}=\hat{a}-\breve{a}$. This shows the importance of being able to evaluate this PDF.

\section{Parameter distribution of the ambiguity residuals}

In general, the GNSS observations are assumed to follow a normal distribution. Hence, also the float estimators are assumed to have a Gaussian distribution. This implies that the marginal PDF of the float ambiguities is given as: 
$f_{\hat{a}}(x)=\frac{1}{\sqrt{\operatorname{det} Q_{\hat{a}}}(2 \pi)^{\frac{1}{2} n}} \exp \left\{-\frac{1}{2}\|x-a\|_{Q_{\hat{a}}}^{2}\right\}$

The ambiguity residuals are defined as:

$\breve{\varepsilon}=\hat{a}-\breve{a}$

These residuals can be used as a measure of the inconsistency between the data and the model. In order to measure the significance of the inconsistency also their probabilistic properties are needed. In practice it is often assumed that the fixed solution $\vec{a}$ is deterministic, so that the residuals simply take the same probability distribution as the float ambiguities. This assumption, however, is not correct. It should only be used if the PDF of the float ambiguities is sufficiently peaked, so that the probability mass in $S_{a}$ is very close to one, as will be shown below.

In Teunissen (2002) and Verhagen and Teunissen (2004) it was shown how the PDF of the ambiguity residuals can be constructed. Here a somewhat different approach will be followed.

Let $\Re \subset R^{n}$ be an arbitrary subset. Then the probability that $\check{\varepsilon} \in \Re$ is given by:

$$
\begin{aligned}
P(\breve{\varepsilon} \in \Re) & =P(\hat{a}-\breve{a} \in \Re) \\
& =\sum_{z \in Z^{n}} P\left(\hat{a}-\breve{a} \in \Re, \hat{a} \in S_{z}\right) \\
& =\sum_{z \in Z^{n}} P\left(\hat{a}-z \in \Re, \hat{a} \in S_{z}\right) \\
& =\sum_{z \in Z^{n}} P\left(\hat{a} \in\{\Re+z\} \cap S_{z}\right) \\
& =\sum_{z \in Z^{n}} \int_{\{\Re+z\} \cap S_{z}} f_{\hat{a}}(x) \mathrm{d} x \\
& =\sum_{z \in Z^{n}} \int_{\Re \cap S_{0}} f_{\hat{a}}(y+z) \mathrm{d} y \\
& =\int_{\Re} \sum_{z \in Z^{n}} f_{\hat{a}}(x+z) s_{0}(x) \mathrm{d} x
\end{aligned}
$$

where for the sixth equality a change of variable, $y=x-z$, was used. The indicator function $s_{0}(x)$ is defined as:

$s_{0}(x)= \begin{cases}1 & \text { if } x \in S_{0} \\ 0 & \text { otherwise }\end{cases}$

Since Eq. 7 holds for any $\Re$, it follows that the PDF of $\breve{\varepsilon}$ is given as:

$$
\begin{array}{r}
f_{\breve{\varepsilon}}(x)=\sum_{z \in Z^{n}} f_{\hat{a}}(x+z) s_{0}(x)=\frac{1}{\sqrt{\operatorname{det} Q_{\hat{a}}}(2 \pi)^{\frac{1}{2} n}} \\
\sum_{z \in Z^{n}} \exp \left\{-\frac{1}{2}\|x-a+z\|_{Q_{\hat{a}}}^{2}\right\} s_{0}(x)
\end{array}
$$

Note that the PDF is only sensitive to the fractional part of $a$, that is the departure from integerness if $a \notin Z^{n}$. But in general it is of course assumed that $a \in Z^{n}$, and then $a$ can be eliminated from Eq. 9 because of the infinite sum over all integers.

If the PDF of $\hat{a}$ is sufficiently peaked, such that all probability mass is in $S_{a}$, we have that:

$f_{\breve{\varepsilon}}(x)=\sum_{z \in Z^{n}} f_{\hat{a}}(x+z) s_{0}(x)=f_{\hat{a}}(x+a) s_{0}(x)$

So, then indeed the PDF of the ambiguity residuals takes the same probability distribution as the float ambiguities, only translated over $-a$.

As was shown in Teunissen (2002) and Verhagen and Teunissen (2004) the joint PDF of $a$ and $\bar{\varepsilon}$ is given by $f_{\breve{\varepsilon}, \vec{a}}(x, z)=f_{\hat{a}}(x+z) s_{0}(x)$. Fig. 1 shows all steps required for the construction of the PDF of the ambiguity residuals in the one-dimensional (1-D) case. The PDF of $\hat{a}$ (top left) is plotted along the $x$-axis, the PMF of $\bar{a}$ (top right) along the $z$-axis, and the joint PDF (top middle) is plotted in the $x z$-plane. Its construction from the marginal PDF and PMF can be seen as follows. First, the parts of the PDF of $\hat{a}$ are sliced out that correspond to all pull-in regions. For the 1-D case, the pull-in regions simply are intervals with length 1 , centered at the integers, $S_{z}=\left\{x \in R|| x-z \mid \leq \frac{1}{2}\right\}$. These slices are then translated along the $z$-axis to the corresponding integers $z$. The joint PDF of $\bar{\varepsilon}$ and $a$ (bottom left) follows from another translation of the slices, but now along the $x$ axis, so that they are all centered at the mean value $x=$ 0 . The PDF of $\varepsilon$ (bottom right) is finally obtained by a summation over $z$, i.e. all slices are again translated along the $z$-axis to the origin.

\section{PDF evaluation}

Unfortunately, it is not possible to evaluate the PDF of the ambiguity residuals exactly because of the infinite sum over all integer vectors in $Z^{n}$. It is therefore required to choose a finite subset $\Theta \subset Z^{n}$ that will give a good approximation. This will be the case if:

$$
\sum_{u \in Z^{n} \backslash \Theta} \frac{1}{\sqrt{\operatorname{det} Q_{\hat{a}}}(2 \pi)^{\frac{n}{2}}} \exp \left(-\frac{1}{2}\|x-a+u\|_{Q_{\hat{a}}}^{2}\right) \approx 0
$$

In order to have a high probability that Eq. 10 holds, the integer set $\Theta$ should be chosen such that the probability

$P\left(\frac{1}{\sqrt{\operatorname{det} Q_{\hat{a}}}(2 \pi)^{\frac{n}{2}}} \exp \left(-\frac{1}{2}\|x-a+z\|_{Q_{\hat{a}}}^{2}\right)>\lambda\right)$

becomes close to one, since then $\lambda$ is so small that there is almost no probability density outside the region 
Fig. 1 The construction of $f_{\breve{s}}(x)$ from $f_{\hat{a}}(x)$ : PDF $f_{\hat{a}}(x)$ (top left); joint PDF $f_{\hat{a}, \vec{a}}(x, z)$ (top center); PMF $P(a=z)$ (top right); joint $\mathrm{PDF} f_{\breve{\varepsilon}, a}(x, z)$

(bottom center); PDF $f_{\breve{\varepsilon}}(x)$ (bottom right)
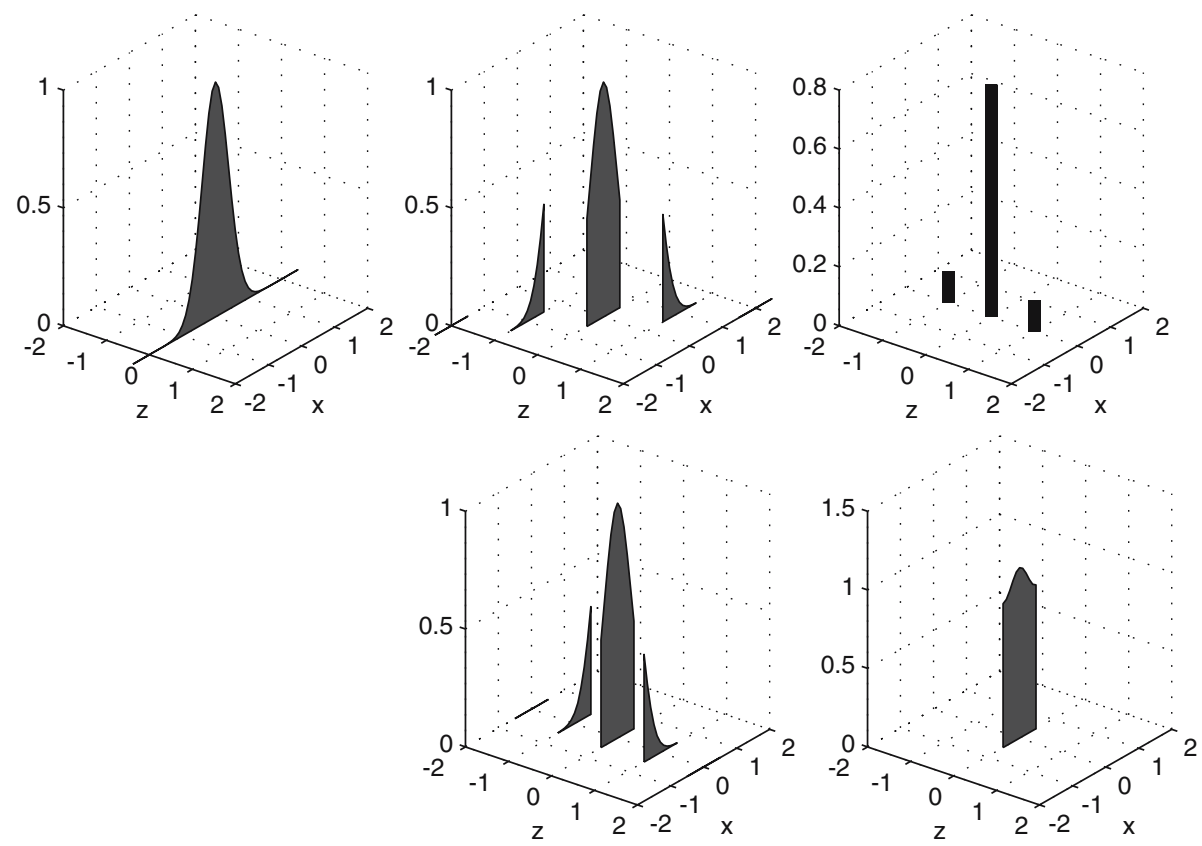

determined by the inequality between the brackets in Eq. 11 , and thus the contribution of all $u \in Z^{n} \backslash \Theta$ to the infinite sum of Eq. 9 can be neglected.

The probability of Eq. 11 can be computed as follows:

$$
\begin{aligned}
& P\left(\frac{1}{\sqrt{\operatorname{det} Q_{\hat{a}}}(2 \pi)^{\frac{n}{2}}} \exp \left(-\frac{1}{2}\|x-a+z\|_{Q_{\hat{a}}}^{2}\right)>\lambda\right) \\
& \quad=P(\|x-a+z\|_{Q_{\hat{a}}}^{2}<\underbrace{-2 \ln \left(\lambda \sqrt{\operatorname{det} Q_{\hat{a}}}(2 \pi)^{\frac{n}{2}}\right)}_{\chi^{2}}) \\
& \quad=P\left(\|\hat{a}-a\|_{Q_{\hat{a}}}^{2}<\chi^{2}\right)
\end{aligned}
$$

This probability can be computed since $\|\hat{a}-a\|_{Q_{\hat{a}}}^{2} \sim \chi^{2}(n, 0)$, where $\chi^{2}(n, 0)$ denotes the central $\chi^{2}$-distribution with $n$ degrees of freedom.

Summarizing, this means that the PDF of the ambiguity residuals can be evaluated as follows:

$f_{\widetilde{\varepsilon}}(x)=\frac{1}{\sqrt{\operatorname{det} Q_{\hat{a}}}(2 \pi)^{\frac{1}{2} n}} \sum_{z \in \Theta} \exp \left(-\frac{1}{2}\|x-a+z\|_{Q_{\hat{a}}}^{2}\right) s_{0}(x)$

with

$\Theta=\left\{z \in Z^{n} \mid\|x+z\|_{Q_{a}}^{2} \leq \chi^{2}\right\}$,

where $a \in Z^{n}$ is eliminated from Eq. 9, and $\chi^{2}$ as defined in Eq. 12. So, the integer set contains all integers $z$ within the ellipsoid centered at -it $\mathrm{x}$ with radius $\chi$. In De Jonge and Tiberius (1996) it is explained how such an integer set can be obtained, using the LAMBDA method.

\section{Results}

Examples

Figure 2 shows $f_{\smile}(x)$ for different values of the standard deviation $\sigma_{\hat{a}}=\sigma$ in the one-dimensional case $(n=1)$. Also the extreme cases, $\sigma=0$ and $\sigma \rightarrow \infty$, are shown. In the first case, an impulse PDF is obtained, in the second case a uniform PDF. Note that the unit of $\sigma$ is cycles.

It can be seen that the PDF becomes peaked if the precision is better, i.e. if $\sigma \downarrow 0$. In that case most of the probability mass of the PDF of $\hat{a}$ is located in the pull-in region $S_{a}$, and the PDFs of $\hat{a}$ and $\breve{\varepsilon}$ would be almost identical. This is the case for $\sigma=0.1$. For $\sigma=0.3$ (right panel) the distribution function becomes flat, and already for $\sigma=1$ the PDF is very close to the uniform distribution. The PDF of the ambiguity residuals may look similar to a normal distribution, but it does not have the infinite tails. Moreover, the corresponding normal PDF of the float ambiguities is always below the PDF of the ambiguity residuals.

Figure 3 shows the contour lines of the PDFs of $\hat{a}$ and $\varepsilon$ obtained for the ILS estimator. The following covariance matrix was used:

$Q_{\hat{a}}=\left(\begin{array}{ll}0.0865 & 0.0364 \\ 0.0364 & 0.0847\end{array}\right)$ 
Fig. 2 PDF of $\breve{\varepsilon}$ for different standard deviations $\sigma$

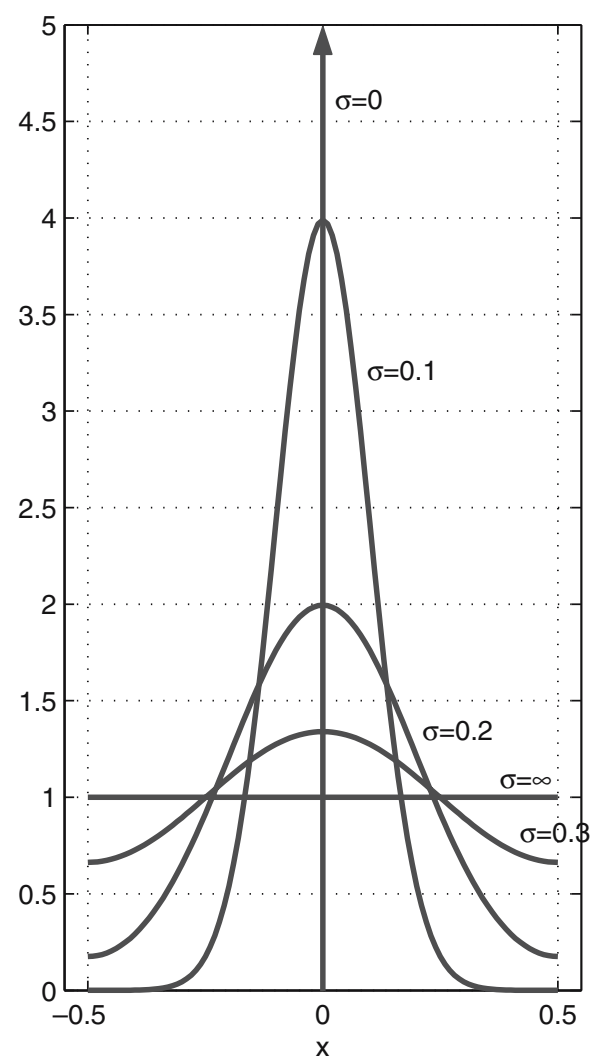

The PDF of the residuals 'fits' the shape of the pull-in region quite well. However, the shape of the PDF near the boundaries of the pull-in region is clearly different from the shape of the PDF of $\hat{a}$. This shows that the fixed ambiguities should not be considered deterministic, since that would result in the assumption that $f_{\breve{s}}(x):=f_{\hat{a}}(x+\breve{a})$. As explained in section 2 this is only true if all the probability mass of $\hat{a}$ is located in the pullin region $S_{a}$. By definition $f_{\breve{\varepsilon}}(x) s_{0}(x) \geq f_{\hat{a}}(x+a) s_{0}(x)$, as can be seen in the figure. Note that only the probability density of $\hat{a}$ within the ILS pull-in region $S_{a=0}$ is shown, but there is also probability mass outside $S_{0}$, whereas all probability mass of $\bar{\varepsilon}$ is within $S_{0}$.

\section{Approximation}

In section 3 it was explained that only an approximation of the PDF of $\check{\varepsilon}$ is possible by replacing the infinite sum over all integers in Eq. 9 by a sum over a finite set of integers. It is investigated here how good the approximations work for different choices of $\lambda$ which determines the integer set, see Eq. 12. For that purpose, 10,000 samples of float ambiguities were generated using simulation for various covariance matrices $Q_{\hat{a}}$. The procedure is as follows. A random generator is used to generate $n$ independent samples from the univariate normal distribution $N(0,1)$, which are collected in a
Fig. 3 Contour lines of the PDFs of $\hat{a}$ (left) and $\breve{\varepsilon}($ right). Only the density within the pullin region $S_{a=0}$ is shown
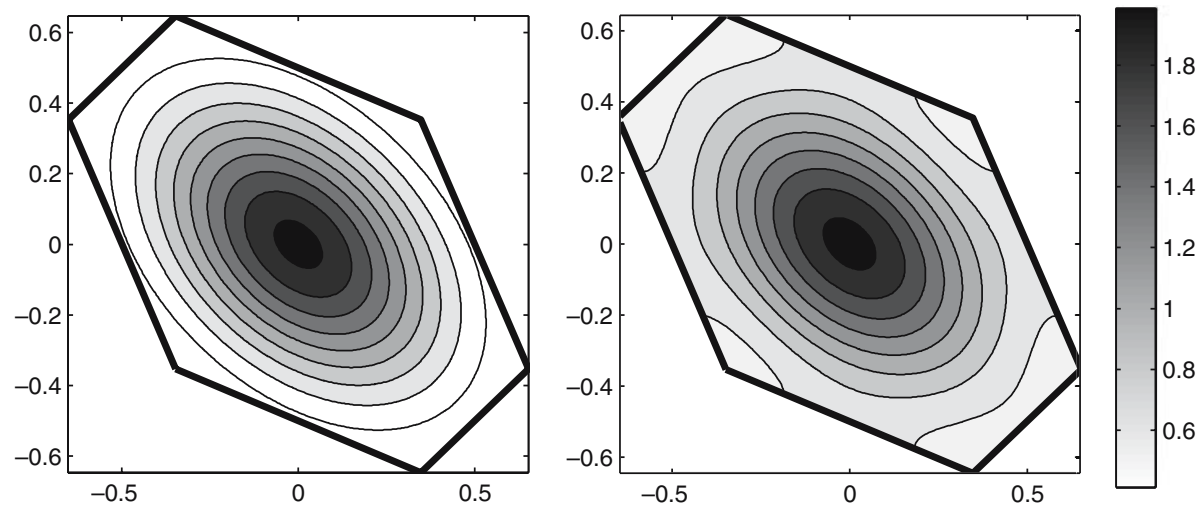
vector $s$. This vector is transformed by means of $\hat{a}=G s$, with $G$ the Cholesky factor of $Q_{\hat{a}}=G G^{T}$. The result is a sample $\hat{a}$ from $N\left(0, Q_{\hat{a}}\right)$, and this sample is used to determine the corresponding ambiguity residuals, so that the PDF of these parameters could be determined.

\section{2-D examples}

The results for the 2-D case are shown in Fig. 4. Besides $Q_{\hat{a}}$ from Eq. 15, also $\frac{1}{4} Q_{\hat{a}}$ and $4 Q_{\hat{a}}$ were used in order to study the effect of higher/lower precision. The horizontal axis gives the value of $\alpha$ :

$\alpha=1-P\left(\|\hat{a}-a\|_{Q_{\hat{a}}}^{2}<\chi^{2}\right)$

See Eq. 12.

The 'correct' values, $f_{\breve{\varepsilon}}^{c}(x)$, were computed for all samples using a very large integer set. Then the differences with the approximations $d f=f_{\breve{\varepsilon}}^{c}(x)-f_{\stackrel{\varepsilon}{\alpha}}^{\alpha}(x)$
Fig. 4 Approximation errors in

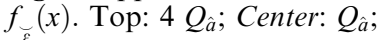
Bottom: $0.25 Q_{\hat{a}}$. Left: Mean errors (dashed) and maximum errors (solid); Right: The mean number (dashed) and maximum number (solid) of integer vectors in the integer set
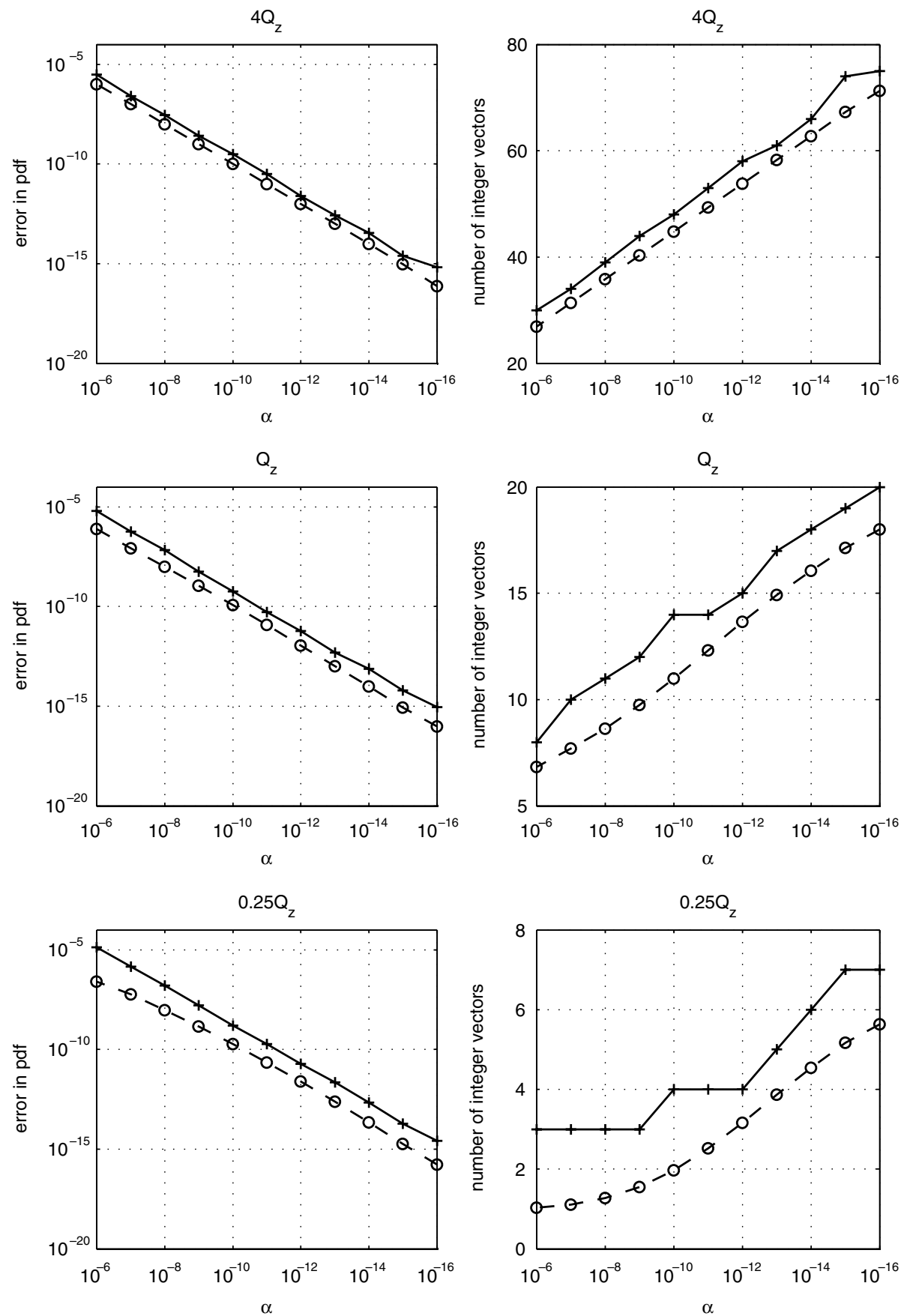
were computed. The top panels show along the vertical axes:

$\begin{array}{ll}y=\frac{d f}{N} & \text { mean error } \\ y=\arg \max _{x}(d f) & \text { maximum error }\end{array}$

$N$ is the number of samples.

It follows that for lower precision - top panels - a large integer set is required in order to get a good approximation. Note that the number of integers in the set $\Theta$ depends on the sample value of $\breve{\varepsilon}$, since the ellipsoidal region is centered at $-\breve{\varepsilon}$.

Geometry-based examples

Samples of float solutions were also generated for four geometry-based, dual-frequency GPS models. The results are summarize in table 1 . These models were set up based on the Yuma almanac for 19-FEB-2002, for two different times and locations (10.05 am, $4^{\circ}$ East, 52 North; and $5 \mathrm{pm}, 52^{\circ}$ East, $4^{\circ}$ North), for which 4 and 6 satellites were visible. Undifferenced standard deviations of $30 \mathrm{~cm}$ and $3 \mathrm{~mm}$ were used for the code and phase observations, respectively. An ionosphere-weighted model was used (Odijk, 2002), and the baseline length was chosen short to medium length by varying the ionospheric standard deviation, $\sigma_{I}$, see table 1 .

For each model, four different integer sets were chosen, based on the choice of $\alpha$, see Eq. 16. The corresponding value of $\lambda$ in Eq. 12 is also given in table 1 . The largest of these sets was chosen such that the error in the approximation of the PDF was small enough to be neglected. The mean and maximum approximation errors were determined based on all samples. The minimum and maximum number of integers in the set $\Theta$ is also given in table 1 .

It follows that the approximation errors have the same order as $\alpha$. So, with $\alpha=10^{-6}$ the errors are small, and the number of integers in the set $\Theta$ is also small enough to guarantee reasonable computation times. This shows that the approach to select a finite integer set gives good approximations of the PDF of the ambiguity residuals.

\section{Summary}

In this contribution the probability density function of the ambiguity residuals was presented. Exact evaluation of the PDF is not possible due to an infinite sum over all integers in $Z^{n}$. Therefore, it is also shown how the PDF can be evaluated.

It follows from the examples that the fixed ambiguity estimator should not be considered deterministic, since there may be a significant difference between the PDF of the float ambiguities and the PDF of the ambiguity residuals. Furthermore, it has been shown that the approximation of the PDF as proposed here gives good results. The user needs to choose the parameter that determines the integer set which is used to replace to sum over all integers in $Z^{n}$. Thereby, one has to choose between higher accuracy (large integer set), or faster computation times (small integer set). But the results indicate that in general a compromise will be possible.

Table 1 Order of the mean and maximum approximation errors in $f_{\breve{\varepsilon}}(x)$ for different models

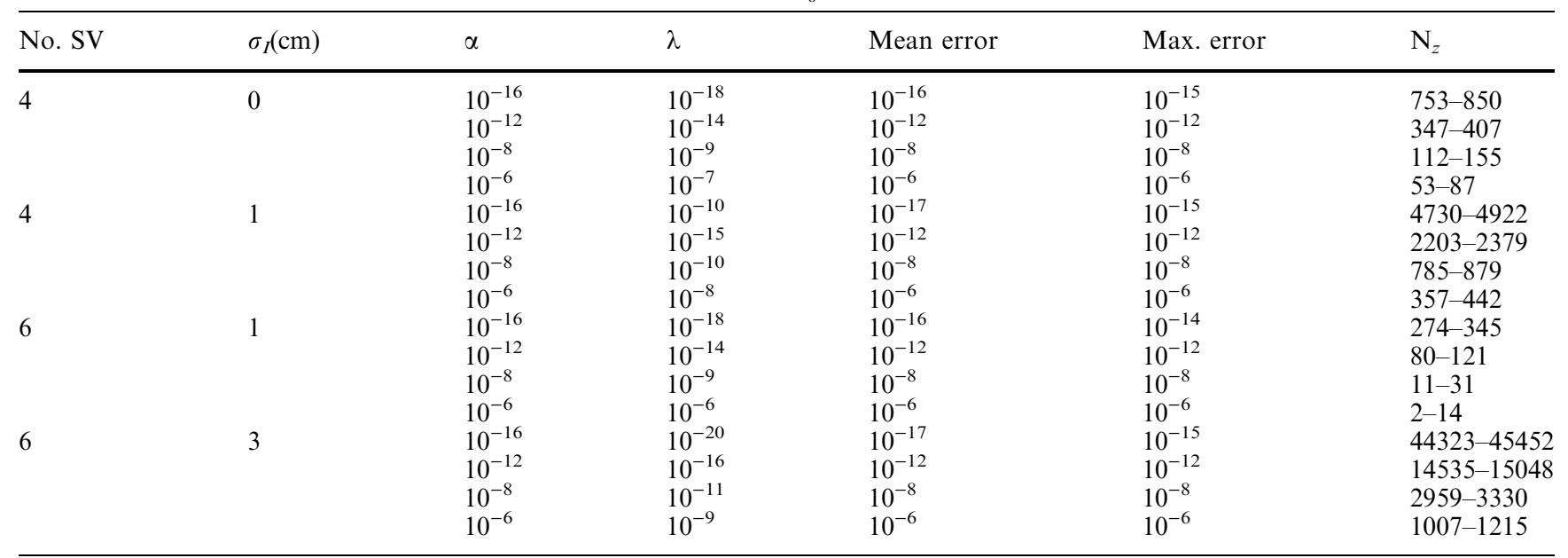

The first two columns show the number of satellites (no. SV), and the ionospheric standard deviation $\left(\sigma_{I}\right) . N_{z}$ equals the minimum and maximum number of integers used 


\section{References}

Boon F, Ambrosius B (1997) Results of real-time applications of the LAMBDA method in GPS based aircraft landings. Proceedings KIS97, pp 339-345

Cox DB, Brading JDW (1999) Integration of LAMBDA ambiguity resolution with Kalman filter for relative navigation of spacecraft. Proceedings ION NTM-99, pp 739-745

De Jonge PJ, Tiberius CCJM (1996) The LAMBDA method for integer ambiguity estimation: implementation aspects. Delft Geodetic Computing Centre, LGR series, no.12, Delft University of Technology, $49 \mathrm{pp}$

Odijk D (2002) Fast precise GPS positioning in the presence of ionospheric delays. Ph.D. thesis, Publications on Geodesy, 52, Netherlands Geodetic Commission, Delft
Peng HM, Chang FR, Wang LS (1999), Attitude determination using GPS carrier phase and compass data, Proceedings ION-NTM-99, pp 727-732

Teunissen PJG (1993) Least squares estimation of the integer GPS ambiguities. In: Invited lecture, section IV theory and methodology, IAG General Meeting, Beijing

Teunissen PJG (1995) The least-squares ambiguity decorrelation adjustment: a method for fast GPS integer ambiguity estimation. J Geodesy 70:65-82

Teunissen PJG (1999) An optimality property of the integer least-squares estimator. J Geodesy, 73, 587-593. DOI $10.1007 / \mathrm{s} 001900050269$

Teunissen PJG (2002) The parameter distributions of the integer GPS model. J Geodesy 76:41-48. DOI 10.1007/ s001900100223
Teunissen PJG (2003a) Integer aperture GNSS ambiguity resolution. Arti Satellites 38(3):79-88

Teunissen PJG (2003b) Towards a unified theory of GNSS ambiguity resolution. J Glob Position Sys 2(1):1-12

Teunissen PJG (2004) Penalized GNSS ambiguity resolution. J Geodesy, 78:235-244. DOI 10.1007/s00190-0040393-2

Teunissen PJG (2005) Optimal integer aperture estimation. Artif Satelli (submitted)

Verhagen S, Teunissen PJG (2004) PDF evaluation of the ambiguity residuals In: Sansò F (ed) V Hotine-Marussi symposium on mathematical geodesy. International Association of Geodesy Symposia, Vol. 127, Springer, Berlin Heidelberg New York 